\title{
Dementia Diagnostics in Primary Care: A Representative 8-Year Follow-Up Study in Lower Saxony, Germany
}

\author{
Lienhard Maeck $^{\mathrm{a}}$ Sebastian Haak ${ }^{\mathrm{b}}$ Anita Knoblauch ${ }^{\mathrm{c}}$ Gabriela Stoppe $^{\mathrm{a}}$ \\ a University Psychiatric Hospitals, Basel, Switzerland; ${ }^{b}$ Klinikum Oldenburg, Oldenburg, and \\ 'Niedersächsisches Landeskrankenhaus, Wunstorf, Germany
}

\section{Key Words}

Primary care • Dementia diagnostics • Memory impairment • Alzheimer's disease $\cdot$ Vascular dementia

\begin{abstract}
Aim: To investigate whether primary-care physicians' competency regarding dementia diagnostics improved from 1993 to 2001. Methods: In a representative follow-up survey 122 out of 170 (71.8\%) family physicians (FPs) were randomly assigned to 2 written case samples presenting patients with slight memory impairment (case 1a: female vs. case 1b: male) and moderate dementia [vascular type (case 2a) vs. Alzheimer's disease (case 2b)]. Potential diagnostic workup was inquired by a structured face-to-face interview. Results: 'Basic' diagnostics like history taking or laboratory investigations were considered in the first place. In case 1, neuropsychological screening was significantly more frequently considered at follow-up (19.3\% in 1993 vs. $31.1 \%$ in 2001 ); it still would have been applied rarely in case 2 (2a: 14.1 vs. $14.8 \%$; 2b: 23.5 vs. $24.6 \%)$. Neuroimaging remained not to be considered as a standard procedure, and only a minority of FPs would have performed a screening for depression (2001: 1a: 6.7\%; 1b: 11.3\%; 2a: 0.0\%; 2b: 1.6\%). Conclusions: With regard to dementia diagnostics in primary care, guideline adherence remained low at follow-up. Structured training efforts aiming at FPs appear to be necessary.
\end{abstract}

Copyright ๑ 2007 S. Karger AG, Basel

\section{KARGER}

Fax +41613061234

E-Mail karger@karger.ch

www.karger.com (c) 2007 S. Karger AG, Basel

$1420-8008 / 08 / 0252-0127 \$ 24.50 / 0$

Accessible online at:

www.karger.com/dem

\section{Introduction}

In the industrialized world and many developing countries, life expectancy is still increasing. As advanced age has to be regarded as the main risk factor for dementia, prevalence rates are supposed to double every 20 years to 81.1 million by 2040 worldwide [1]. As for the situation in Germany, likewise, the number of about 1 million of demented people today will double within the next 4 decades [2]. The resulting economic burden on society is immense, as at least for the largest subgroup of primary degenerative dementia of the Alzheimer type (DAT), treatment options still remain limited. In general, dementia should be diagnosed early, as potentially curable causes of a dementia syndrome - like depression and side-effects of polypharmacy - benefit from early treatment. Moreover, there is evidence that in case of primary degenerative dementia, early treatment will result in slowing the course of disease and delay nursing home placement significantly [3]. In Germany and other countries, medical care for the elderly is particularly provided by family physicians (FPs) $[4,5]$. Usually, the assumption of this task is in accordance with their professional selfconception [6-8]. However, early diagnosis of dementia is a challenging subject, as the first symptoms are rather non-specific. Last but not least the physicians' subjective expectations towards the therapeutic impact of treatment options in dementia may also affect diagnostic consider-

Prof. Dr. med. Gabriela Stoppe

University Psychiatric Hospitals

Wilhelm Klein-Strasse 27

CH-4025 Basel (Switzerland)

Tel. +41 61325 5646, Fax +41 61325 5582, E-Mail gabriela.stoppe@upkbs.ch 
ations. For example, earlier studies could demonstrate that both the rate of diagnosis and individual case management depend on the doctors' knowledge as well as on his/her tenor with regard to dementia. Even more, they also unravelled a high prevalence of nihilistic attitudes [6-11]. It is therefore not amazing that during recent years several investigators showed deficits in FPs' skills with respect to the diagnosis and treatment of dementia [1215].

In a representative survey of FPs in Lower Saxony dating from 1993, our study group applied standardized exemplary case vignettes in personal face-to-face interviews in the physicians' practices. Two written case samples were presented to 145 general practitioners and primary-care internists. One of them described a patient with slight memory complaints without any vascular risk factors or somatic comorbidity, the other one a patient suffering from moderate dementia from either vascular or Alzheimer type, respectively, and common somatic disorders of late life [16]. The physicians' considerations regarding their potential diagnostic management were recorded and statistically evaluated. Above all, 'classical' diagnostic procedures were considered [detailed direct anamnesis, physical examination, blood examination, blood pressure measurement, electrocardiogram (ECG)]. A minority of FPs decided on taking a thorough indirect anamnesis, neuropsychological testing, screening for depression or neuroimaging.

In the following years, the rollout of specifically acting cholinesterase inhibitors for the exclusive therapy of DAT attracted considerable public attention and was accompanied by substantial training efforts aiming at physicians involved in the diagnosis and treatment of dementia. Due to their relatively high price, cost-benefit ratio has been a matter of controversial discussion from the very beginning. Nevertheless, as dementia increasingly got an issue at stake for physicians, geriatric contents became integrated into the education of future FPs. The present follow-up survey was conducted to verify the resulting assumption that primary-care skills regarding the diagnostic management of cognitive decline in the elderly might have improved since 1993.

\section{Materials and Methods}

The detailed methods as well as the text of the applied sample case histories have been published recently [17]. According to the list of physicians obtained by the Board of Physicians of Lower Saxony, 2 trained investigators (A.K. and S.H.) addressed all FPs maintaining private practices in the cities of Göttingen, Nort- heim and their closer vicinity. To ensure satisfying collaboration, the FPs were asked for a standardized face-to-face interview with respect to their diagnostic workup of cognitive decline in elderly patients. By this mode of questioning, standardized open questions could be posed without disclosing any answer categories; moreover, answers induced by the questionnaire could be avoided. The term 'dementia' was not explicitly given to keep the study focus as blinded as possible. The interview was documented in written form, for the most part by ticking off prepared answer categories. In case the request was rejected, our investigators tried to get the statistical data of the physician and the practice by phone, in order to evaluate any differences in the group of participants and non-participants [physicians' age and gender, year of licence to practise medicine, medical qualification, date of launching the medical practice, practice location, joint practice (yes/no), list size, special interest in geriatric psychiatry (yes/no), typical clientele, estimated percentage of pensioners among clientele, estimated number of demented patients per year]. Physicians consenting to an interview likewise answered the statistical questionnaire as a first step. Then, to each participating doctor, 2 randomly assigned case vignettes were presented by the interviewers $(1 \mathrm{a}+2 \mathrm{a}$ or $1 \mathrm{a}+2 \mathrm{~b}$ or $1 \mathrm{~b}+2 \mathrm{a}$ or $1 \mathrm{~b}+2 \mathrm{~b})$. To ensure comparability, the case vignettes were almost identical to those presented in our preliminary survey dating from 1993 [16].

Only differing in the patients' gender, cases $1 \mathrm{a}$ and $1 \mathrm{~b}$ described a person with slight memory impairment of 6 months duration; according to ICD-10 criteria, a beginning dementia syndrome may be suggested [18]. In 1993, the patient was female and, in half of the cases, expressed the wish for a drug. Since no influence on the resulting prescription rate could be detected, the latter topic was excluded in our survey from 2001 [19]. On the other hand, as another investigation on the primary-care management of depression unravelled gender differences with regard to the patients' and physicians' gender, we decided to allow for gender differences in our follow-up [20].

Cases $2 \mathrm{a}$ and $2 \mathrm{~b}$ both described a female patient suffering from moderate dementia and common disorders typical of old age, such as adiposity, hypercholesterolaemia, type II diabetes, arthrosis, myocardial insufficiency and arterial hypertension. Case 2a pointed to a primarily vascular aetiology of the dementia syndrome, whereas $2 \mathrm{~b}$ described the characteristics of DAT. Compared to the situation in 1993, case 2 was modified with regard to drug therapy, as an ACE inhibitor replaced the treatment with digitalis and a diuretic.

After studying the case presentations, the participating physicians were asked standardized questions regarding their potential diagnostic management (average duration of interview: $15 \mathrm{~min}$ ). The answers obtained were categorized to facilitate the interpretation of the data. In this paper, responses to the following questions will be discussed:

- What kind of diagnostics would you arrange for every patient (please also refer to 'basic' procedures)?

- Would you refer the respective patient to a specialist (psychiatrist or neurologist)?

In 1993 we included a small number of neuropsychiatrists $(\mathrm{n}=14)$ in private practice serving as orientation for the group of specialists. Although in 2001 a larger group was investigated, due to the preceding small case number, only follow-up data for the FPs will be reported and discussed in the following. 
Statistical Analysis

The $\chi^{2}$ test was used to compare a- and b-versions of the case vignettes, male versus female, and junior versus senior physicians. As each doctor got 1 version of sample 1 and 1 version of sample 2, with respect to both cases, the responses could not be considered as independent. To ensure comparability of 1- and 2versions, we employed McNemar's test. As the same geographic region was investigated, McNemar's test was also applied to compare the answers obtained in 1993 with those of our follow-up. Remarkably, only a minority of physicians spontaneously remembered the former investigation, although many of them had taken part in the previous survey. $\chi^{2}$ was compared to a $\chi^{2}$ distribution with 1 degree of freedom and a confidence level of $95 \%$; thus, the results were regarded as significant at a level of $p<0.05$.

\section{Results}

In the year 2001, in Göttingen, Northeim and vicinity, a total of 196 FPs were acting in private practice (1993: 226). Of this population, 26 physicians had to be excluded for reasons like treating an inappropriate clientele. Of the remaining 170 physicians, 122 (71.8\%; 1993: $83.2 \%)$ agreed to take part in our survey. The groups of participants and non-participants differed significantly regarding the type of private practice, as $66.4 \%$ of the participants but only $43.8 \%$ of the rejecting physicians were practising alone. Age, gender, list size, additional qualifications and rural vs. urban locations were without influence on readiness in participating so that the criteria for a representative study were met. Among the group of participating FPs, the percentage of female physicians rose significantly from $30.3 \%$ in 1993 to $44.3 \%$ in 2001 ( $p<0.05)$. In contrast, the portion of FPs stating a special interest in geriatric psychiatric topics markedly decreased from $66.9 \%$ in 1993 to $35.2 \%$ in 2001 (p < $0.05)$.

FPs' diagnoses with respect to both sample case histories have been previously discussed [17]. In summary, we found a prevailing vascular concept of dementia genesis as well as a tendency of underdiagnosing probable DAT (cases 1a/1b: DAT: $11.0 \%$ in 1993 vs. $26.2 \%$ in 2001; vascular dementia: $2.1 \%$ in 1993 vs. $17.2 \%$ in 2001). Concomitant medication was only exceptionally considered as a contributing factor to a dementia syndrome (cases 2a/2b: $4.4 \%$ in 1993 vs. $2.5 \%$ in 2001). Regarding cases $1 \mathrm{a} / 1 \mathrm{~b}$, physicians more than 50 years of age showed a significantly lower diagnostic awareness as they tended to diagnose more frequently no disease $(32.1$ vs. $3.1 \% \mathrm{p}<$ $0.006)$ and less frequently DAT (10.7 vs. $37.5 \%$; $<<0.069)$ [17].

Dementia Diagnostics in Primary Care

\section{Case 1: Diagnostics}

In 2001, there were no significant differences regarding the parallel versions $1 \mathrm{a}$ and $1 \mathrm{~b}$. In both sample case vignettes, laboratory studies were mentioned most frequently as diagnostic tool (1993: $81.7 \%$ vs. $2001: 77.4 \%$ ). They were followed by taking the detailed direct anamnesis (71.7 vs. $80.6 \%$ ), a physical examination (56.7 vs. $69.4 \%$ ), an ECG (60.0 vs. $53.3 \%$ ) and blood pressure measurement (50.0 vs. $48.4 \%$ ). The inquiry of an indirect anamnesis as well as performing neuropsychological tests were mentioned by about one third of FPs, neuroimaging by slightly more than $10 \%$ and screening for depression by 6.7 vs. $11.3 \%$, respectively. An electroencephalogram (EEG) was taken into consideration only exceptionally (1.7 vs. $4.8 \%)$

With respect to the patient's gender, no significant differences became apparent in the course of time. The cumulated results for sample case vignettes $1 \mathrm{a}$ and $1 \mathrm{~b}$ are given in table 1. In comparison to 1993, at follow-up, neuropsychological screening was considered significantly more often (1993: 19.3\% vs. 2001: 31.1\%; $p=0.037$ ). During the same period of time, both ECG and taking the blood pressure significantly lost importance (ECG: 69.0 vs. $56.6 \%$; $p=0.049$; blood pressure measurement: 83.4 vs. $49.2 \%$; $\mathrm{p}<0.001$ ). The small percentage of FPs considering neuroradiological investigations, mainly cranial computer tomography (CCT), remained stable at followup (CCT: 12.4 vs. 11.5\%). Like in 1993, magnetic resonance imaging (MRI) was only exceptionally mentioned in 2001 (0.7 vs. $0.8 \%)$.

\section{Case 1: Referral to a Specialist?}

More than $50 \%$ of the participating doctors would have sent the patient to a specialist (1993: $54.1 \%$ vs. $2001: 57.4 \%$ ). There were no significant differences regarding the parallel versions 1a and 1b; likewise, the FPs' age, gender, list size, additional qualifications or practice location were without influence on the potential referral behaviour.

\section{Cases 2: Diagnostics}

Case 2 was subdivided in vignette $2 \mathrm{a}$ (moderate vascular dementia) and $2 \mathrm{~b}$ (moderate DAT).

Case 2a: Like in 1993, basic diagnostics like the investigation of a blood sample (1993: $80.8 \%$ vs. $2001: 82.0 \%$ ), measuring the blood pressure ( 85.9 vs. $62.3 \%$ ), taking the detailed history (1993: $74.4 \%$ vs. 2001: 68.8\%), ECG (74.4 vs. $60.6 \%)$ and performing a physical examination (62.8 vs. $60.6 \%$ ) were given priority (table 2). Compared to the situation in 1993, at follow-up, EEG (47.4 vs. $11.5 \%$; p < $0.001)$ and taking the blood pressure ( 85.9 vs. $62.3 \%$; $\mathrm{p}=$

Dement Geriatr Cogn Disord 2008;25:127-134 
Table 1. Diagnostic procedures considered in the time course (case vignette 1 )

\begin{tabular}{|c|c|c|c|c|c|}
\hline \multirow[t]{2}{*}{ Diagnostic procedures } & \multicolumn{2}{|c|}{$1993(n=145)$} & \multicolumn{2}{|c|}{$2001(n=122)$} & \multirow[t]{2}{*}{$\mathrm{p}$} \\
\hline & $\mathrm{n}$ & $\%$ & $\mathrm{n}$ & $\%$ & \\
\hline Direct anamnesis & 108 & 74.5 & 93 & 76.2 & n.s. \\
\hline \multicolumn{6}{|l|}{ Laboratory studies } \\
\hline Blood count & 102 & 70.3 & 97 & 79.5 & n.s. \\
\hline Renal values & 100 & 69.0 & 82 & 67.2 & n.s. \\
\hline Electrolytes & 97 & 66.9 & 71 & 58.2 & n.s. \\
\hline Blood glucose & 92 & 63.4 & 75 & 61.5 & n.s. \\
\hline Liver values & 90 & 62.1 & 71 & 58.2 & n.s. \\
\hline Blood lipids & 95 & 65.5 & 57 & 46.7 & 0.003 \\
\hline Thyroid hormones & 69 & 47.5 & 66 & 54.1 & n.s. \\
\hline Blood sedimentation reaction & 96 & 66.2 & 37 & 30.3 & $<0.001$ \\
\hline Differential blood count & 6 & 4.1 & 6 & 4.9 & n.s. \\
\hline Haemodynamometry & 121 & 83.4 & 60 & 49.2 & $<0.001$ \\
\hline ECG & 100 & 69.0 & 69 & 56.6 & 0.049 \\
\hline Physical examination & 88 & 60.7 & 77 & 63.1 & n.s. \\
\hline Indirect anamnesis & 33 & 22.8 & 37 & 30.3 & n.s. \\
\hline Neuropsychological screening & 28 & 19.3 & 38 & 31.1 & 0.037 \\
\hline Doppler ultrasonography & 19 & 13.1 & 25 & 20.5 & n.s. \\
\hline EEG & 32 & 22.1 & 4 & 3.3 & $<0.001$ \\
\hline CCT & 18 & 12.4 & 14 & 11.5 & n.s. \\
\hline Depression screening & 0 & 0 & 11 & 9.0 & n.s. \\
\hline Chest X-ray & 3 & 2.1 & 1 & 0.8 & n.s. \\
\hline MRI & 1 & 0.7 & 1 & 0.8 & n.s. \\
\hline
\end{tabular}

$\mathrm{CCT}=$ Cranial computer tomography; $\mathrm{MRI}=$ magnetic resonance imaging; $\mathrm{n} . \mathrm{s} .=$ not significant.
0.003) were considered significantly less frequently; there was also an obvious trend towards less generous use of neuroimaging, especially CCT (43.6 vs. $29.5 \%$ ).

Case $2 b$ : Like in case $2 \mathrm{a}$, the above-mentioned classical diagnostic procedures were given in the first place (table 3). Compared to 1993, at follow-up significantly fewer FPs decided for blood pressure measurement (77.8 vs. 54.1\%; $\mathrm{p}=0.005)$ and EEG (40.7 vs. $6.6 \%$; $<0.001)$. In comparison to case $2 \mathrm{a}$, in case $2 \mathrm{~b}$, neuropsychological testing was considered more frequently (2001: $2 \mathrm{a}$ : $14.8 \%$ vs. 2 b: $24.6 \%)$. This finding was shown to remain stable in the course of time (1993: 14.1 vs. 23.5\%). Likewise, performing a CCT was considered significantly more frequently in case $2 \mathrm{a}$, which was true for 1993 and follow-up (1993: 2a: $43.6 \%$ vs. 2 b: $27.2 \%$; 2001: 29.5 vs. $14.8 \%$ ). Like in case $2 \mathrm{a}$, with respect to neuroimaging, there was a trend towards a more restrictive handling. Taken together, at both times of our survey, laboratory investigations, EEG and neuroimaging were considered more frequently in case $2 \mathrm{a}$, whereas neuropsychological screening was rather mentioned in case $2 b$. For both cases, with the exception of thyroid function test, any significant changes in time course concern a reduction in the employment of the specific diagnostic instrument (ECG, EEG, blood pressure measurement, certain laboratory parameters).

\section{Cases 2: Referral to a Specialist?}

In both parallel versions of case 2 , at follow-up, about $80 \%$ of the FPs would have sent their patient to a specialist, which represents a significant increase of about $15 \%$ each compared to the situation in 1993 ( $\mathrm{p}<0.05)$. As a consequence, a referral would have been significantly more frequently considered in the case of moderate dementia than in the patient with slight memory impairment (case 1).

\section{Discussion}

As about $70-80 \%$ of the patients with cognitive disorders address their FPs in the first place, the latter face an important task in providing early recognition and adequate management of dementia. Beginning with the approval of tacrine in 1995, the treatment options of DAT 
Table 2. Diagnostic procedures considered in the time course (case vignette $2 \mathrm{a}$ )

\begin{tabular}{|c|c|c|c|c|c|}
\hline \multirow[t]{2}{*}{ Diagnostic procedures } & \multicolumn{2}{|c|}{$1993(n=78)$} & \multicolumn{2}{|c|}{$2001(n=61)$} & \multirow[t]{2}{*}{$\mathrm{p}$} \\
\hline & $\mathrm{n}$ & $\%$ & $\mathrm{n}$ & $\%$ & \\
\hline \multicolumn{6}{|l|}{ Laboratory studies } \\
\hline Blood glucose & 63 & 80.8 & 50 & 82.0 & n.s. \\
\hline Blood count & 58 & 74.4 & 50 & 82.0 & n.s. \\
\hline Renal values & 59 & 75.6 & 47 & 77.0 & n.s. \\
\hline Electrolytes & 58 & 74.4 & 41 & 67.2 & n.s. \\
\hline Blood lipids & 57 & 73.1 & 38 & 62.3 & n.s. \\
\hline Liver values & 50 & 64.1 & 42 & 68.9 & n.s. \\
\hline Blood sedimentation reaction & 54 & 69.2 & 18 & 29.5 & $<0.001$ \\
\hline Thyroid hormones & 12 & 15.4 & 30 & 49.2 & $<0.001$ \\
\hline Vitamins & 7 & 9.0 & 3 & 4.9 & n.s. \\
\hline Blood coagulation & 0 & 0 & 5 & 8.2 & n.s. \\
\hline Differential blood count & 3 & 3.8 & 2 & 3.3 & n.s. \\
\hline Haemodynamometry & 67 & 85.9 & 38 & 62.3 & 0.003 \\
\hline Direct anamnesis & 58 & 74.4 & 42 & 68.8 & n.s. \\
\hline ECG & 58 & 74.4 & 37 & 60.6 & n.s. \\
\hline Physical examination & 49 & 62.8 & 37 & 60.6 & n.s. \\
\hline CCT & 34 & 43.6 & 18 & 29.5 & n.s. \\
\hline Doppler ultrasonography & 23 & 29.5 & 19 & 31.1 & n.s. \\
\hline EEG & 37 & 47.4 & 7 & 11.5 & $<0.001$ \\
\hline Indirect anamnesis & 21 & 26.9 & 17 & 27.9 & n.s. \\
\hline Neuropsychological screening & 11 & 14.1 & 9 & 14.8 & n.s. \\
\hline Chest X-ray & 3 & 3.8 & 2 & 3.3 & n.s. \\
\hline MRI neurocranium & 3 & 3.8 & 2 & 3.3 & n.s. \\
\hline Echocardiography & 0 & 0 & 2 & 3.3 & n.s. \\
\hline Cerebrospinal puncture & 0 & 0 & 1 & 1.7 & n.s. \\
\hline Depression screening & 0 & 0 & 0 & 0.0 & n.s. \\
\hline
\end{tabular}

have substantially expanded. Along with considerable marketing and training efforts of the pharmaceutical industry aiming at FPs, dementia experienced an emergence of positive representations in public interest [21]. In view of the growing number of consultations due to memory deficits in the recent years, we were interested to see whether FPs' diagnostic management of dementia might have improved within the period between 1993 and 2001.

In 1993, based on a representative survey of general practitioners in Lower Saxony, Germany, we used written case vignettes to examine FPs' diagnostic considerations in patients with mild cognitive decline as well as moderate dementia. Among others, we could demonstrate a considerable underdiagnosis of dementia in 1993 [16]. At follow-up in 2001, dementia was considered significantly more often; however, we found a persisting overestimation of vascular aetiology and underdiagnosis of probable DAT [17].

Dementia Diagnostics in Primary Care
In the following, methodological proceedings will not be subject of discussion in detail, as they have been delineated earlier [16-19]. Employing standardized case vignettes in a personal interview results in high participation rates, and, as long as the study focus is blinded as well as possible, allows to reliably re-investigate changes in time course [22]. As pre- as well as postgraduate education of FPs in Germany follows comparable standards all over the country, there are no plausible causes to assume regional differences in medical competency. Together with a high participation rate and the absence of significant differences between the groups of participating and non-participating physicians, our follow-up study can be considered as representative. Since we addressed all FPs of a certain region at 2 distinct points in time (1993 and 2001), in both surveys, the groups of FPs were not identical. Those who were taking part twice could only rarely remember their participation 8 years before. To our knowledge, our survey presents the first representative

Dement Geriatr Cogn Disord 2008;25:127-134 131 
Table 3. Diagnostic procedures considered in the time course (case vignette $2 \mathrm{~b}$ )

\begin{tabular}{|c|c|c|c|c|c|}
\hline \multirow[t]{2}{*}{ Diagnostic procedures } & \multicolumn{2}{|c|}{$1993(\mathrm{n}=81)$} & \multicolumn{2}{|c|}{$2001(n=61)$} & \multirow[t]{2}{*}{$\mathrm{p}$} \\
\hline & $\mathrm{n}$ & $\%$ & $\mathrm{n}$ & $\%$ & \\
\hline \multicolumn{6}{|l|}{ Laboratory studies } \\
\hline Blood glucose & 67 & 82.7 & 39 & 63.9 & 0.019 \\
\hline Blood count & 63 & 77.8 & 41 & 67.2 & n.s. \\
\hline Renal values & 66 & 81.5 & 34 & 55.7 & 0.002 \\
\hline Electrolytes & 65 & 80.2 & 32 & 52.5 & $<0.001$ \\
\hline Blood lipids & 66 & 81.5 & 30 & 49.2 & $<0.001$ \\
\hline Liver values & 59 & 72.8 & 29 & 47.5 & 0.004 \\
\hline Blood sedimentation reaction & 62 & 76.5 & 13 & 21.3 & $<0.001$ \\
\hline Thyroid hormones & 26 & 32.1 & 32 & 52.5 & 0.023 \\
\hline Vitamins & 10 & 12.3 & 6 & 9.8 & n.s. \\
\hline Differential blood count & 3 & 3.7 & 6 & 9.8 & n.s. \\
\hline Blood coagulation & 0 & 0 & 7 & 11.5 & n.s. \\
\hline Direct anamnesis & 56 & 69.1 & 42 & 68.9 & n.s. \\
\hline Haemodynamometry & 63 & 77.8 & 33 & 54.1 & 0.005 \\
\hline ECG & 57 & 70.4 & 34 & 55.7 & n.s. \\
\hline Physical examination & 54 & 66.7 & 32 & 52.5 & n.s. \\
\hline Doppler ultrasonography & 21 & 25.9 & 18 & 29.5 & n.s. \\
\hline Neuropsychological screening & 19 & 23.5 & 15 & 24.6 & n.s. \\
\hline EEG & 33 & 40.7 & 4 & 6.6 & $<0.001$ \\
\hline Indirect anamnesis & 15 & 18.5 & 16 & 26.2 & n.s. \\
\hline CCT & 22 & 27.2 & 9 & 14.8 & n.s. \\
\hline Echocardiography & 0 & 0 & 10 & 16.4 & n.s. \\
\hline Chest X-ray & 7 & 8.6 & 4 & 6.6 & n.s. \\
\hline MRI & 2 & 2.5 & 3 & 4.9 & n.s. \\
\hline Depression screening & 0 & 0 & 1 & 1.6 & n.s. \\
\hline Cerebrospinal puncture & 0 & 0 & 0 & 0.0 & n.s. \\
\hline
\end{tabular}

n.s. = Not significant. follow-up data with regard to FPs' management of cognitive decline in the elderly.

Like in 1993, 4 case vignettes were applied in 2001 (1a, $1 \mathrm{~b}, 2 \mathrm{a}, 2 \mathrm{~b})$, cases $1 \mathrm{a}$ and $1 \mathrm{~b}$ only differing in the patients' gender. Case 1 describes a patient complaining about progressive concentration and memory deficits for 6 months as well as an increasing emotional irritability. The ICD-10 criteria of dementia are not fulfilled; nevertheless, in the absence of any concomitant disease or medication, a prodromal syndrome of dementia as well as depression should be considered as underlying aetiology. Cases 2a and $2 \mathrm{~b}$ describe a multimorbid demented patient who exhibits a vascular risk profile and is on continuous drug treatment. Whereas case $2 \mathrm{a}$ refers to a primary vascular aetiology, $2 \mathrm{~b}$ points to typical DAT. Additionally, in both case vignettes, the 2 main causes of treatable dementia should be taken into diagnostic consideration (adverse effects of medication, systemic disorder). With regard to their cooperation in surveys, in 2001 physicians more fre- quently complained about cost pressure, overwork and slackening of motivation than in 1993. Accordingly, the readiness to take part in our study decreased slightly from $83.2 \%$ in 1993 to $71.8 \%$ in 2001 ; however, it remained on a comparatively high level.

Despite a variety of national and international guidelines for the diagnosis and treatment of dementia, they generally emphasize the necessity of a clinical diagnosis [direct and indirect anamnesis including possible polypharmacy; psychiatric and physical/neurological examination including screening for depression, laboratory studies and ECG; neuropsychological testing/screening; cerebral imaging if suspicion of (degenerative) dementia remains justified; additional procedures dependent on previous results] [23]. In case 1, the patients' history - together with other basic procedures - was regarded as the main diagnostic tool both in 1993 and 2001. However, at follow-up in 2001, it ranks behind the laboratory investigations, which were taken into consideration in the fore- 
most place. One explanation might be that primary-care physicians, having known their patients and their histories for years, thought taking a new detailed history was not necessary. Neuropsychological screenings and tests serve as an objective measure for cognitive deficits and are especially useful in mild forms of dementia; in 2001, they were considered significantly more frequently (31.1 vs. 19.3\%). Technical procedures such as neuroimaging were still not regarded as standard procedures, and examination of cerebrospinal fluid was not mentioned at all. Furthermore, only a minority of primary-care physicians would have performed a screening for depression, even though depression is a main differential diagnosis in mild cognitive disorder. EEG was not regarded as an important diagnostic tool and was suggested significantly less often in 2001 than in 1993 (3.3 vs. 22.1\%). This is in accordance with international guidelines, as EEG is almost exclusively part of German guidelines [23].

Concerning case 2 no distinctive changes are found in the time course. However, thyroid function tests would be performed more often in 2001, namely by approximately half of the physicians. While further laboratory function tests were suggested less often in case 2b, EEG and taking the blood pressure were thought of less in both cases than in 1993. As in case 1, taking the patient's history by relatives or friends, neuroimaging as well as screening for depression were considered only rarely. Especially the percentage of CCT was reduced compared to 1993, even though the number of MRI did not increase correspondingly. In contrast to case 1 , there was no change concerning neuropsychological tests; in case $2 \mathrm{a}$ roughly $14 \%$ of the physicians would have suggested such an examination. This is especially noteworthy, as primary-care physicians' representatives in Germany recom- mend the use of neuropsychological screening tests, which is also in accordance with international guidelines $[23,24]$. On the other hand, current investigations point out that most primary-care physicians do not regard tests practicable, as the time it takes to do the tests is not compatible with their budget and schedule [13, 25].

With regard to dementia, here, we present the first representative empirical study on the development of physicians' diagnostic management in the time course. Compared to 1993, international as well as national dementia guidelines remained to be accomplished inconsequently in 2001 [23], as neuropsychological screening/testing, neuroimaging, screening for depression or even taking an indirect history were still considered too rarely (i.e. neuropsychological screening by less than one third of FPs). As 'cheap' neuropsychological screening tests would have been applied rather than 'expensive' neuroimaging, budgetary pressure might considerably influence FPs' diagnostic workup. Moreover, apart from FPs' potential nihilistic attitudes towards dementia [6-11], uncertainties with respect to their own diagnostic skills as well as the potential need to communicate a highly 'unwanted' diagnosis might forward FPs' avoidance behaviour regarding diagnostic workup. Nevertheless it seems appropriate that the training of (primary-care) physicians should integrate guideline-adherent contents of geriatric psychiatry to a larger extent.

\section{Acknowledgement}

This study was funded by the German Federal Ministry of Health and Social Affairs.

\section{References}

$>1$ Ferri CP, Prince M, Brayne C, Brodaty H, Fratiglioni L, Ganguli M, Hall K, Hasegawa K, Hendrie H, Huang Y, Jorm A, Mathers C, Mezenes PR, Rimmer E, Scazufca M; Alzheimer's Disease International: Global prevalence of dementia: a Delphi consensus study. Lancet 2005;366:2112-2117.

$>2$ Bickel H: Dementia in advanced age: estimating incidence and health care costs. Z Gerontol Geriatr 2001;34:108-115.

3 Geldmacher DS, Provenzano G, McRae T, Mastey V, Ieni JR: Donepezil is associated with delayed nursing home placement in patients with Alzheimer's disease. J Am Geriatr Soc 2003;51:937-944.
4 Small GW, Rabins PV, Barry PP, Buckholtz NS, DeKosky ST, Ferris SH, Finkel SI, Gwyther LP, Khachaturian ZS, Lebowitz BD, McRae TD, Morris JC, Oakley F, Schneider LS, Streim JE, Sunderland T, Teri LA, Tune LE: Diagnosis and treatment of Alzheimer disease and related disorders: consensus statement of the American Association for Geriatric Psychiatry, the Alzheimer's Association, and the American Geriatrics Society. JAMA 1997;278:1363-1371.

5 Mayer KU, Baltes PB (eds): The Berlin Aging Study. Berlin, Akademie Verlag, 1996.
6 Turner S, Iliffe S, Downs M, Wilcock J, Bryans M, Levin E, Keady J, O’Carroll R: General practitioners' knowledge, confidence and attitudes in the diagnosis and management of dementia. Age Ageing 2004;33:461467.

7 Iliffe S, Manthorpe J, Eden A: Sooner or later? Issues in the early diagnosis of dementia in general practice: a qualitative study. Fam Pract 2003;20:376-381.

8 Waldorff FB, Almind G, Mäkelä M, Møller S, Waldemar G: Implementation of a clinical dementia guideline: a controlled study on the effect of a multifaceted strategy. Scand J Prim Health Care 2003;21:142-147. 
-9 Cody M, Beck C, Shue VM, Pope S: Reported practices of primary care physicians in the diagnosis and management of dementia. Aging Ment Health 2002;6:72-76.

10 Van Hout H, Vernooij-Dassen M, Bakker K, Blom M, Grol R: General practitioners on dementia: tasks, practices and obstacles. Patient Educ Couns 2000;39:219-225.

-11 Riedel-Heller SG, Schork A, Matschinger H, Angermeyer MC: The role of referrals in diagnosing dementia at the primary care level. Int Psychogeriatr 1999;11:251-262.

12 Boustani M, Callahan CM, Unverzagt FW, Austrom MG, Perkins AJ, Fultz BA, Hui SL, Hendrie HC: Implementing a screening and diagnosis program for dementia in primary care. J Gen Intern Med 2005;20:572-577.

13 Boise L, Neal MB, Kaye J: Dementia assessment in primary care: results from a study in three managed care systems. J Gerontol A Biol Sci Med Sci 2004;59:621-626.

14 Löppönen MK, Isoaho RE, Räihä IJ, Vahlberg TJ, Loikas SM, Takala TI, Puolijoki H, Irjala KM, Kivelä SL: Undiagnosed diseases in patients with dementia - a potential target group for intervention. Dement Geriatr Cogn Disord 2004; 18:321-329.
5 Olafsdottir M, Foldevi M, Marcusson J: Dementia in primary care: why the low detection rate? Scand J Prim Health Care 2001;19: 194-198.

16 Stoppe G, Sandholzer H, Staedt J, Winter S, Kiefer J, Kochen MM, Rüther E: Diagnosis of dementia in primary care: results of a representative study in Lower Saxony, Germany. Eur Arch Psychiatry Clin Neurosci 1994; 244:278-283.

17 Maeck L, Haak S, Knoblauch A, Stoppe G: Early diagnosis of dementia in primary care: a representative eight-year follow-up study in Lower Saxony, Germany. Int J Geriatr Psychiatry 2006;22:23-31.

18 World Health Organization: Tenth Revision of the International Classification of Diseases, chapt. V (F): Mental and behavioural disorders (including disorders of psychological development). Clinical descriptions and diagnostic guidelines. Geneva, WHO, 1991.

9 Stoppe G, Sandholzer H, Staedt J, Winter S, Kiefer J, Rüther E: Reasons for prescribing cognition enhancers in primary care: results of a representative survey in Lower Saxony, Germany. Int J Clin Pharmacol Ther 1995; 33:486-490.
20 Stoppe G, Sandholzer H, Huppertz C, Duwe $\mathrm{H}$, Staedt J: Gender differences in the recognition of depression in old age. Maturitas 1999;32:205-212.

21 Ngatcha-Ribert L: Alzheimer disease and society: an analysis of its social representation. Psychol Neuropsychiatr Vieil 2004;2:49-66.

22 Holt WS, Mazzucca SA: A written case simulation of osteoarthritis as a predictor of prescribing behaviour among family practitioners. Acad Med 1992;67:414-415.

23 Müller U, Wolf H, Kiefer M, et al: A systematic comparison of national and international dementia guidelines. Fortschr Neurol Psychiatr 2003;71:285-295.

24 Bund Deutscher Allgemeinmediziner (BDA): Manual Demenz, ed 2. Emsdetten, BDA, 1999.

25 Pentzek M, Fuchs A, Abholz HH: The attitudes of general practitioners regarding dementia - cognitive, affective, and external components. Nervenheilkunde 2005;24: 499-506. 